\title{
LIPID PEROXIDATION AND ANTIOXIDANT CONSUMPTION AS EARLY MARKERS OF NEUROSURGERY-RELATED BRAIN INJURY IN CHILDREN
}

\author{
${ }^{1}$ M.Piastra, ${ }^{1,5}$ E.Caresta, ${ }^{3}$ L.Massimi, ${ }^{1,4,5}$ D. De Luca, ${ }^{1}$ G.Conti, ${ }^{1}$ E.Luca, ${ }^{6}$ S.Eaton \\ ${ }^{1}$ Paediatric ICU and Institute of Anesthesia/Intensive Care, Emergency and Intensive Care Department, \\ ${ }^{3}$ Paediatric Neurosurgery, Dept of Neurosurgery; ${ }^{4}$ Institute of Clinical Biochemistry \\ Catholic University Medical School, "A.Gemelli" Hospital, Rome, Italy; ${ }^{5}$ Neonatal and Paediatric ICU, Paris-Sud \\ University, Paris, France; ${ }^{6} \mathrm{UCL}$ Great Ormond Street Institute of Child Health, London, UK
}




\begin{abstract}
Background and Aims

Lipid peroxidation represents a marker of secondary brain injury both in traumatic and in nontraumatic conditions-as in major neurosurgical procedures-eventually leading to brain edema amplification and further brain damage. Malondialdehyde (MDA), a lipid peroxidation marker, and ascorbate, a marker of antioxidant status, can represent early indicators of this process within the cerebrospinal fluid (CSF). We hypothesized that changes in cerebral lipid peroxidation can be measured ex vivo following neurosurgery in children.
\end{abstract}

\title{
Methods
}

Thirty-six children (M:F = 19/17, median age 32.9 months; IQR 17.6-74.6) undergoing neurosurgery for brain tumor removal were admitted to the pediatric intensive care unit (PICU) in the postoperative period with an indwelling intraventricular catheter for intracranial pressure monitoring and CSF drainage. Plasma and CSF samples were obtained for serial measurement of MDA, ascorbate, and cytokines.

\section{Results}

An early brain-limited increase in lipid peroxidation was measured, with a significant increase from baseline of MDA in CSF ( $p=0.007)$ but not in plasma. In parallel, ascorbate in CSF decreased $(p=0.05)$. Systemic inflammatory response following brain surgery was evidenced by plasma IL-6/IL8 increase ( $p 0.0022$ and 0.0106 , respectively). No correlation was found between oxidative response and tumor site or histology (according to World Health Organization grading). Similarly, lipid peroxidation was unrelated to the length of surgery (mean $321 \pm 73 \mathrm{~min}$ ), or intraoperative blood loss (mean $20.9 \pm 16.8 \%$ of preoperative volemia, $44 \%$ given hemotransfusions). Median PICU stay was 3.5 days (IQL range $2-5.5 \mathrm{~d}$.), and postoperative ventilation need was $24 \mathrm{~h}$ (IQL range 20$61.5 \mathrm{~h}$ ). The elevation in postoperative MDA in CSF compared with preoperative values correlated significantly with postoperative ventilation need $(P=0.05, r 2$ 0168), while no difference in PICU stay was recorded.

\section{Conclusions}

Our results indicate that lipid peroxidation increases consistently following brain surgery, and it is accompanied by a decrease in antioxidant defences; intraventricular catheterization offers a unique chance of oxidative process monitoring. Further studies are needed to evaluate whether monitoring post-neurosurgical oxidative stress in CSF is of prognostic utility.

\section{Keywords}

Pediatric neurosurgery Pediatric intensive care Antioxidant activity Lipid peroxidation Brain injury 


\section{Introduction.}

Neurosurgery involving brain tissue remains an invasive procedure regardless of whether it is performed electively or emergently. Some neurosurgical interventions, such as those for brain stem and spinal cord pathologies, are intrinsically linked to postoperative neurological deficits and may lead to serious neurological injuries regardless of how carefully the operation is carried out [1]. Indeed, most of these procedures are associated with unavoidable injury inflicted on the functional and normal brain while dissecting or removing the pathological tissue(s). Many injuries are consistent with predetermined cortical incisions to access any deeper pathological tissue, retraction of brain lobes or hemispheres, intraoperative bleeding [2], and thermal injury due to electrocoagulation. Even minimally invasive endoscopic procedures can lead to brain tissue damage and complications [3].

Brain edema is the clinical hallmark of all types of brain injury, possibly leading to intracranial hypertension and secondary brain damage; therefore, the elucidation of mechanisms leading to increased central nervous system (CNS) water content is of major importance. Infants and children are considered at risk of brain swelling in response to noxious stimuli. Strategies to minimize neuroinflammation and reactive brain edema may be of vital importance also in the perioperative period $[4,5]$. At present, an effective neuroprotective treatment against surgical/iatrogenic brain injury is greatly awaited. The aim of our study is to explore the perioperative oxidative stress leading to lipid peroxidation through the examination of intracranial cerebrospinal fluid (CSF) over time.

\section{Materials and Methods}

Following local institutional review board approval, the study was carried out as part of an investigational protocol regarding neuroprotection in the perioperative period of brain tumor removal. A total of 36 children were included in this non-randomized open prospective trial. Children were 2-8 yr old, American Society of Anesthesiologists physical status I or II, and scheduled for general anesthesia and outpatient elective surgery for intracranial neoplasm.

All the children received dexamethasone $0.5 \mathrm{mg} / \mathrm{kg} /$ day due to the presence of peritumoral edema in the perioperative period of brain neoplasm removal. 
An intraventricular drainage was electively placed at the beginning of the surgical procedure because of hydrocephalus and maintained (for intracranial pressure monitoring or CSF drainage) during the early postoperative course. The intraventricular drain was removed as soon as clinically indicated. CSF and plasma samples were taken preoperatively and 12, 24, and $36 \mathrm{~h}$ postoperatively.

\section{Lipid Peroxidation Marker Measurements}

Plasma and CSF malondialdehyde (MDA) levels, a marker of lipid peroxidation, were measured by the high-performance liquid chromatography. Plasma MDA was measured as previously described using $25 \mu \mathrm{l}$ plasma [6]. For CSF, $100 \mu \mathrm{l}$ CSF was incubated with $2.5 \mu \mathrm{l}(0.2 \% \mathrm{w} / \mathrm{v})$ butylated hydroxytoluene in ethanol plus $375 \mu \mathrm{l} 1 \%(\mathrm{v} / \mathrm{v})$ phosphoric acid for $10 \mathrm{~min}$, and $345 \mu \mathrm{l} 0.22 \%$ thiobarbituric acid and incubated for $60 \mathrm{~min}$ at $100{ }^{\circ} \mathrm{C}$ for $60 \mathrm{~min}$. After cooling, the MDAthiobarbituric acid derivative was extracted with $400 \mu$ l butanol. To an autosampler vial, $250 \mu \mathrm{l}$ butanol phase was transferred, butanol evaporated off under $\mathrm{N} 2$ at $80{ }^{\circ} \mathrm{C}$, and the MDAthiobarbituric acid derivative redissolved in $150 \mu$ buffer and analyzed by HPLC as for plasma [6]. CSF ascorbate was measured by a spectrophotometric assay as follows: $0.3 \mathrm{ml}$ ice-cold $10 \%$ trichloroacetic acid was added to $0.2 \mathrm{ml} \mathrm{CSF}$ and the mixture kept on ice for $30 \mathrm{~min}$. Following this, the sample was centrifuged $(13,000 \mathrm{rpm} \times 5 \mathrm{~min})$ and $0.37 \mathrm{ml}$ supernatant removed, and $0.074 \mathrm{ml}$ of dinitrophenylhydrazine reagent added, consisting of $0.4 \mathrm{~g}$ thiourea, $0.05 \mathrm{~g}$ CuSO4.5H2O, $3.0 \mathrm{~g}$ 2,4dinitrophenylhydrazine in $100 \mathrm{ml} 9.0 \mathrm{~N} \mathrm{H} 2 \mathrm{SO} 4$. Following mixing, $0.555 \mathrm{ml}$ 65\% H2SO4 was added, and the samples measured at $520 \mathrm{~nm}$ in a spectrophotometer [7]. Cytokines (L-8, IL-1ß, IL-6, IL-10, TNF, and IL-12p70) were analyzed by a multiplex bead method (BD ${ }^{\text {TM }}$ Cytometric Bead Array Human Inflammation Kit) according to the manufacturer's instructions.

\section{Anesthesia Protocol}

General anesthesia was induced through a face mask with sevoflurane (6-8\%) and 02 in noncooperating patients, by means of thiopentone $(3-4 \mathrm{mg} / \mathrm{Kg}$ ) or propofol $(2 \mathrm{mg} / \mathrm{Kg}$ ) in the older children. Two cannulae were positioned into large-size peripheral veins (mostly large right saphenous vein) together with a central venous line in the internal jugular vein through echo-guided procedure. Heart rate (HR), invasive arterial blood pressure (IABP), central venous pressure (CVP), superior caval vein mixed venous saturation (scvSvO2), pulse oximetry (SpO2), EtHA (end tidal halogenated agents), EtCO2, spirometry, peripheral versus core temperature, urine output and, acid-base status (including arterial lactate concentration), and $\mathrm{Hb} / \mathrm{Hct} /$ platelet were systematically 
monitored. Body temperature was maintained by hot air warming mattress; fluid infusions were warmed thermostatically. Muscle relaxation was obtained by cisatracurium besylate $(0.1 \mathrm{mg} \mathrm{Kg-1}$ bolus, 2 mcg Kg-1 min-1 continuous infusion). Endotracheal intubation was performed using cuffed tracheal tubes. General anesthesia was maintained by sevoflurane (MAC 1.5) and remifentanil (0.25-0.5 mcg Kg-1 h-1) according to patient demand, while ventilation was given through Datex Aestiva (Datex, Helsinki) on a volume-controlled mode.

The following metabolic data $(\mathrm{Na}+, \mathrm{Cl}-, \mathrm{BUN}$, creatinine, $\mathrm{ALT}$, bilirubin, $\mathrm{CPK}$, lactate, $\mathrm{pH}$, base excess, bicarbonate) were assessed preoperatively, at the end of surgery and 24,48 , and $72 \mathrm{~h}$ post-surgery.

Fluid intake was tailored according to hemodynamic and metabolic demand except in patients with interstitial cystitis lesions, where fluid infusion was maintained at a rate of $4 \mathrm{ml} / \mathrm{Kg} / \mathrm{h}$ until dural opening. No hypotonic or glucose solutions were administered during surgery. A tight control of fluids input/output and related hemodynamic parameters (IABP, CVP, HR) was performed: During surgery, every effort was made to maintain hemodynamic stability; in clinical practice, aggressive volume management with balanced solutions, colloids (hydroxyethyl starch), and blood transfusions (if Htc lower than 21\%). Colloid solutions (up to $20 \mathrm{~mL} \mathrm{Kg-1} \mathrm{B.W.} \mathrm{of} \mathrm{hydroxyethyl} \mathrm{starch,} \mathrm{then} 5 \%$ Human Albumin) were given to replace blood loss and maintain patient volemia. When $\mathrm{O} 2$ delivery was deemed not adequate to support tissue metabolism (on the basis of clinical and laboratory data, as scvSvO2 and lactate trend), packed red blood cells (PBRC) were transfused to restore Hct. Fresh-frozen plasma (10-15 mL kg-1) was given when prothrombin time (PT) and activated partial thromboplastin time (aPTT) were 1.5 longer than normal values, according to the international guidelines [8]. ATIII concentrate was administered when its plasma level was $<70 \%$, whereas platelet concentrates were given if platelet count fell below $50.000 \mu \mathrm{L}-1$ (Fig. 1). At the end of surgery, all patients were admitted to the Pediatric Intensive Care Unit (PICU) for postoperative care.

\section{Hematological and Coagulative Assessment}

Hemoglobin $(\mathrm{Hb})$, hematocrit $(\mathrm{Hct}), \mathrm{PT}$, aPTT, fibrinogen concentration, and platelet count were measured preoperatively, at the end of surgery and 24,48 , and $72 \mathrm{~h}$ post-surgery. Red cell volume was calculated as follows: estimated red cell volume $(E R C V)=$ estimated blood volume $\times$ Hct/100] Transfused blood volume was estimated as follows: estimated red cell volume transfused 
$(E R C T)=0.6$ (mean hematocrit of packed red cells units) $\times$ PBRC, where PBRC is the transfused volume of packed blood red cells.

We then considered blood loss using the two following formulae: estimated red cell volume loss $(E R C L)=[($ preoperative $E R C V$ - postoperative ERCV $)+E R C T] ; E R C L$ ratio $=E R C L /$ preoperative ERCV.

PICU transfusions in the early postoperative period (within $72 \mathrm{~h}$ from admission) were also recorded. Blood replacement was aimed to maintain hematocrit between 21 and 27\%, with a CVP within 6 and $8 \mathrm{cmH} 2 \mathrm{O}$.

\section{Statistics}

Postoperative samples were compared with the preoperative samples by paired t-tests (for MDA and ascorbate, which were normally distributed) or Wilcoxon matched-pairs tests (cytokines, which were not normally distributed). In order to correct for multiple comparisons, a Bonferroni cutoff of significance was calculated ( 4 comparisons), so that $p<0.05 / 4$, i.e., $p=0.0125$ was considered as significant. 


\section{Results}

\section{Patient Demographics}

The study population included 36 patients, $M / F=19 / 17$, median age of 32.9 months (IQR 17.674.6), and median body weight $14 \mathrm{~kg}$ (IQR 10-21). All patients were affected by posterior cranial fossa neoplasms; 30 patients underwent primary removal and 6 patients had relapsing tumor surgery. Patient demographics and main diagnoses are summarized in Table 1.

\section{Intraoperative Blood Loss}

Median blood loss, as evaluated by means of calculated red blood cell (RBC) volume loss, was $18.1 \%$ (IQE 8.4;28.4) of preoperative value. Blood transfusions were received by $16 / 36$ patients (44\%) with a mean volume of $33 \pm 52 \mathrm{~mL} \mathrm{kg-1}$ packed RBC. Hydroxyethyl starch was given at $14.6 \pm 7.1 \mathrm{~mL} \mathrm{kg-1}$ (Table 1).

\section{Metabolic Data}

All patients received glucose-free infusions during the whole operative time. All patients were treated preoperatively with steroids (dexamethasone $0.5 \mathrm{mg} / \mathrm{kg} / \mathrm{day}$ ). Preoperative glucose level was $89.4 \pm 21.0 \mathrm{mg} / \mathrm{dL}$, whereas PICU admission glycemia was $137.2 \pm 60.0 \mathrm{mg} / \mathrm{dL}$. Median calculated glucose stress index (GSI, calculated as GSI = [postoperative glycemia/preoperative glycemia] $\times$ surgery duration [min]/100) was 4.75 (Table 2, Fig. 2) [9, 10].

\section{Postoperative PICU Stay and Complications}

Median PICU stay in the postoperative period was 3.5 days (IQR 2.0-5.7), whereas ventilatory support was needed for a median time of $24 \mathrm{~h}$ (IQR 20-70). No bleeding complications were recorded; 5 patients had a Staphylococcal CSF infection in the postoperative period (PICU + Ped neurosurgical ward), while 2 patients experienced de novo seizures (Table 1 ).

\section{CSF Lipoperoxidation Markers and Antioxidant Reserve}

An early brain-limited oxidative burst was documented with a significant increase in MDA in CSF ( $p=0.002$ comparing CSF sample after intraventricular catheter placement but before mass debulking with postoperative sample) but not in plasma (Fig. 1). CSF MDA values rapidly returned to pre-debulking levels ( $p=n$.s. compared with pre-debulking). There was no correlation between MDA levels in CSF and plasma $(r 2=0.04)$. There was a short-lived fall in CSF ascorbate levels postoperatively, and the fall and recovery in CSF ascorbate simultaneously occurred with the increases in CSF MDA levels (Fig. 1). Although there was no evidence for a systemic increase in lipid peroxidation markers, there was evidence for a systemic inflammatory response, as indicated by 
increases in plasma IL-6 and IL-8 ( $p=0.0039$ for each, Fig. 3). There were no significant changes in IL-12p70, TNF-a, IL-10, or IL-1b (data not shown). No correlation was found between oxidative response and tumor site or histology (WHO grading). Similarly, neither surgery length (mean $321 \pm 73 \mathrm{~min}$ ) nor blood loss were related to the early oxidative response.

\section{Clinical Outcome Measures}

No early postoperative mortality was recorded. Reported postoperative complications were unrelated both to intraoperative blood loss and to oxidative phenomena. As relevant outcome measures, median PICU stay was 3.5 days (IQL range 2-5.5 d.) and postoperative ventilation need was $24 \mathrm{~h}$ (IQL range 20-61.5 h). Postoperative-to-preoperative CSF MDA ratio showed a significant correlation with postoperative ventilation need $(P=00.05, r 20.168)$, while no correlation with PICU stay was recorded. Differently, GSI, a metabolic distress marker, was correlated with PICU stay $(r$ 0347; p 0.024) and not with mechanical ventilation requirement (Fig. 4).

\section{Discussion}

In this study, we have demonstrated an early brain-limited postoperative increase in lipid peroxidation and an associated decrease in antioxidant status. Both events, namely lipid peroxidation and antioxidant decrease, may represent a threat for secondary brain injury, as a potential early cause for brain edema amplification and further neurological damage. In many CNS diseases, such as traumatic brain injury (TBI), stroke, hydrocephalus, and post-hemorrhagic and post-ischemic brain damage, lipid peroxidation has been demonstrated to play a key role in brain damage [11]. Even CNS infection pathophysiology has been demonstrated to involve oxidative stress $[12,13]$.

Also traumatic brain injury, which is probably among the most studied CNS acute illnesses in childhood, is characterized by a marked and progressive compromise of the antioxidant defenses and free radical-mediated lipid peroxidation $[14,15]$. These products lead to neuronal damage by promoting lipid peroxidation, protein breakdown, and DNA damage which in turn lead to cellular apoptosis, endothelial injury, and BBB permeability [16].

The issue of clinical neuroprotection against oxidative brain injury in neurosurgery has rarely been addressed previously due to lack of any studies in humans and to the unavailability of an appropriate animal model. However, the TBI model has been largely studied and good results in terms of protective effects in mild and severe brain injury have been obtained with alpha tocopherol [17] and with melatonin (when administered $<2 \mathrm{~h}$ post-trauma) [18]. Moreover, a demonstration has been provided that a beneficial effect on oxidative stress may be induced by moderate hypothermia 
in the early post-trauma period [19]. The authors concluded that CSF monitoring through oxidation markers could be valuable to assess the effect of therapies on oxidative stress after TBI in pediatric patients. The observation of an early peak in F2-isoprostane levels (oxidative marker) suggested the possible need for early application of therapies targeting some aspects of oxidative stress after TBI, such as lipid peroxidation [20]

A key issue to be considered during neurosurgical interventions is that there is always an element of unavoidable brain injury that results from the procedure itself due to the unique nature of the nervous system. During neurosurgery, similarly to TBI, several factors that include either direct brain damage (incision, electrocautery, hemorrhage) or secondary brain injury (edema, hypoxia, neuroflogosis) may cause cerebral tissue damage leading to brain dysfunction and neuronal death. The biochemical events involved in this process include energy failure, membrane depolarization, production of oxygen free radicals, and eventually lipid peroxidation $[4,5]$.

In our study, we have interestingly shown that brain-specific increase in lipid peroxidationevaluated into the CSF through serial sampling-exists soon after surgical injury, since no significant MDA or ascorbate variations exist in systemic plasma samples obtained by contemporary collection despite a marked postoperative inflammatory response (witnessed by IL-6 and IL-8 plasma levels increase). The issue of brain-limited oxidative event has been debated in comparison with the available literature: In our opinion, an extensive BBB breakage is only present in severely inflamed brain, as in meningitis-meningoencephalitis patients $[12,13]$. Therefore, most papers dealing with MDA increase in plasma refer to diffusely involved endothelium. We addressed previous studies regarding traumatic brain injury due to similarities between this condition and our setting [14, 15]; therefore, we still believe that a compartmentalization remains between inside and outside the brain in children undergoing brain tumor surgery. This may explain why MDA levels showed marked changes into the LCR and minimal or absent changes into the plasma.

The previous literature demonstrated that lipid peroxidation markers are nearly undetectable in the absence of intracranial disorders [16], whereas the relationship between cancer and oxidative stress has been deeply investigated [20]; hence, cancer cells are certainly under high levels of oxidative stress in comparison with normal cells [21]. Some reports exist about the increased lipid peroxidation also in the plasma of patients with many CNS illnesses, including brain tumors [22].

The aim of the study was not limited to the measurement of basal CSFMDA levels in such a limited sample of children with brain tumor. In fact, MDA basal levels could have been influenced by 
confounding factors as the length of steroid/antiedema therapy, hydrocephalus severity and tension [23], time from diagnosis, etc. More interestingly, we documented an early increase in lipid peroxidation -through CSFMDA serial measurements - as a result of surgical procedure, since basal levels were detected at the time of intraventricular catheter placement, before any manipulation of the tumor and healthy surrounding brain tissue.

In our patients, no correlation has been found neither with tumor histotype nor with grading, leading to the conclusion that it could be a phenomenon strictly related to the neurosurgical brain injury. Of clinical interest, in our experience, we found a correlation between the extent of MDA increase and the postoperative ventilation requirement. No correlation, on the contrary, has been found between lipoperoxidation and PICU stay, which has been related to the glycemic/metabolic stress in this study according to previous experiences of the same study group [8]. As ventilatory support requirement may represent a more specific measure of neurological impairment, MDAratio can help to identify a patient subset with increased intensive care requirements. In the previous literature, the outcome of children admitted to intensive care in the postoperative period of brain tumor removal has been related to intraoperative blood loss and to some tumor-related issues [2, 24]; more recently, a role for metabolic stress response has been described by our study group [9, 10]. Therefore, MDAratio can be considered as a possible prognostic marker of clinical outcome in neurosurgical procedures.

A role for antioxidants neuroprotection in children undergoing neurosurgery can be hypothesized [25], as it occurs for brain trauma patients. The presence of an intraventricular catheter could easily allow the determination of lipoperoxidation markers and its correlation with clinical events both inside the PICU and thereafter. Moreover, a more favorable time window for neuroprotectants may be hypothesized in these patients, due to the availability of preemptive antioxidant treatment.

\section{Study Limitations}

We have to acknowledge the limitations of our study. First, the patient sample is limited and does not allow to make conclusions about the absolute oxidation products levels and their clinical meaning; second, a preoperative evaluation of the anatomical and radiological features of the neoplasms was not performed, as it was not considered relevant for this study. Moreover, given the differences in the surgical mass debulking and postoperative hydrocephalus drainage, also postoperative intracranial pressure was not deemed as a reliable marker of intracranial edema or postoperative damage. Dexamethasone treatment is constantly given to our patients as antiedema 
agent, in the perioperative period of brain neoplasm removal. If a dexamethasone-related effect exists, there are no differences among patients and different time points.

\section{Conclusions}

Lipid peroxidation assessment ex vivo may represent a new tool that may help for diagnosing and monitoring treatments adopted to control secondary brain damage. A successful therapeutic intervention for 'surgically induced brain injury' may result in significant benefits for patients and healthcare organizations. Both events may be studied bedside, by directly assessing lipoperoxidation markers in the CSF using a simple intraventricular catheter. Oxidative stress assessment can be a more reliable predictive marker of intensive care requirement in comparison with previously adopted intraoperative blood loss and metabolic stress markers.

\section{Notes}

\section{Author Contribution Statement}

Marco Piastra MD (M.P.), Elena Caresta MD (E.C.), Giorgio Conti MD (G.C.), and Simon Eaton MD (S.E.) designed the study, analyzed the data, and wrote the first draft of the paper; designed the study and wrote the first draft of the paper; Luca Massimi MD (L.M.), Enzo Picconi MD (E.P.), and Ersilia Luca MD (E.L.) recruited the patients and collected the data; Tony Christian Morena MD (T.C.M.) is the corresponding author, and also recruited the patients and collected the data.

\section{Conflict of interest}

The author(s) declare that they have no competing interests.

\section{Ethical approval/informed consent}

The study was approved by the Institutional Review Board of the Universita Cattolica del Sacro Cuore. Participation in the survey implies informed consent from the participants.

\section{Source of support}

The author(s) declare that they have no competing interest or financial support to acknowledge. 
TABLE. 1 Demographics and clinical data of patients undergoing posterior cranial fossa neoplasm removal

\begin{tabular}{|c|c|}
\hline Patients & $\mathrm{N}=36$ \\
\hline Age, median (IQR) & $32.9(17.6 ; 74.6)$ months \\
\hline Body Weight, median (IQR) & $14(10 ; 21) \mathrm{kg}$ \\
\hline $\mathrm{M} / \mathrm{F}$ & $19 / 17(1,11)$ \\
\hline \multicolumn{2}{|l|}{ Neoplasm Histotype } \\
\hline -medulloblastoma/PNET & $\mathrm{n}=11 \quad$ (WHO IV) \\
\hline -theratoid rhabdoid/malignant teratoma & $\begin{array}{ll}\mathrm{n}=4 & (\mathrm{WHO} I \mathrm{IV})\end{array}$ \\
\hline -ependymoma & $\mathrm{n}=2 \quad$ (WHO III) \\
\hline -ependymoma low grade & $\mathrm{n}=2 \quad$ (WHO II) \\
\hline -pylocitic astrocytoma & $\mathrm{n}=10 \quad(\mathrm{WHO} \mathrm{I})$ \\
\hline -choroid plexus papilloma & $\mathrm{n}=2 \quad(\mathrm{WHO} \mathrm{I})$ \\
\hline -ganglioglioma & $\mathrm{n}=3 \quad(\mathrm{WHO} \mathrm{I})$ \\
\hline \multicolumn{2}{|l|}{ Outcome data } \\
\hline -Blood Loss (as ERCVL\%) median (IQR) & $18.1(8.4 ; 28.4)$ \\
\hline - Transfusions: n.pts (\%); median value (mL) & $\mathrm{n}=16(44.4 \%) ; 190(110 ; 222)$ \\
\hline crystalloids $\mathrm{mL} \mathrm{kg}^{-1}$ & $54(41 ; 75)$ \\
\hline colloids $\mathrm{mL} \mathrm{kg}^{-1}$ & $15(11 ; 28)$ \\
\hline - HES infusion $\mathrm{ml} \mathrm{kg}^{-1}$ & $15(9 ; 17)$ \\
\hline - Surgery duration & $330(246 ; 367) \min$ \\
\hline - Mechanical Ventilation TIME & $24(20.3 ; 69.3)$ hours \\
\hline - PICU stay & $3.5(2.0 ; 5.8)$ days \\
\hline \multicolumn{2}{|l|}{ Postoperative Complications: } \\
\hline - seizures & 3 \\
\hline - CSF infections & 4 \\
\hline - persistent hydrokephalus & 7 \\
\hline - tracheostomy & 1 \\
\hline
\end{tabular}

\section{Metabolic/oxidation markers (median; IQR)}

Basal CSFMDA

Postoperative (1)

Postoperative (2)

Postoperative(3)

DeltacsFMDA (post/preoperative)

Basal CSFAscorbate

Postoperative (1)

Postoperative (2)

Postoperative (3)

Basal PLASMAIL-6

Postoperative (1)

Postoperative (2)

Basal PLASMAlL-8

Postoperative (1)

Postoperative (2)

PREOPGlucose

PICU-ADMGlucose

Glucose Stress Index (GSI)
$0.069(0.045 ; 0.129)$

$1.00(0.66 ; 1.45)$

$0.99(0.60 ; 1.35)$

$1.16(0.66 ; 1.45)$

$1.64(1.25 ; 2.28)$

$165.9(112.1 ; 211.6)$

$135.3(80.4 ; 158.0)$

$124.3(86.1 ; 166.5)$

107.0 (76.2; 169.7)

$0.0(0.0 ; 6.5)$

$45.9(15.5 ; 18.9)$

$16.6(9.5 ; 30.9)$

9.0(4.6; 14.6)

$37.3(23.2 ; 60.0)$

22.0(16.6; 34.7)

$90.5(75.7 ; 108)$

$122(109.8 ; 147.3)$

$4.75(3.64 ; 5.77)$ 

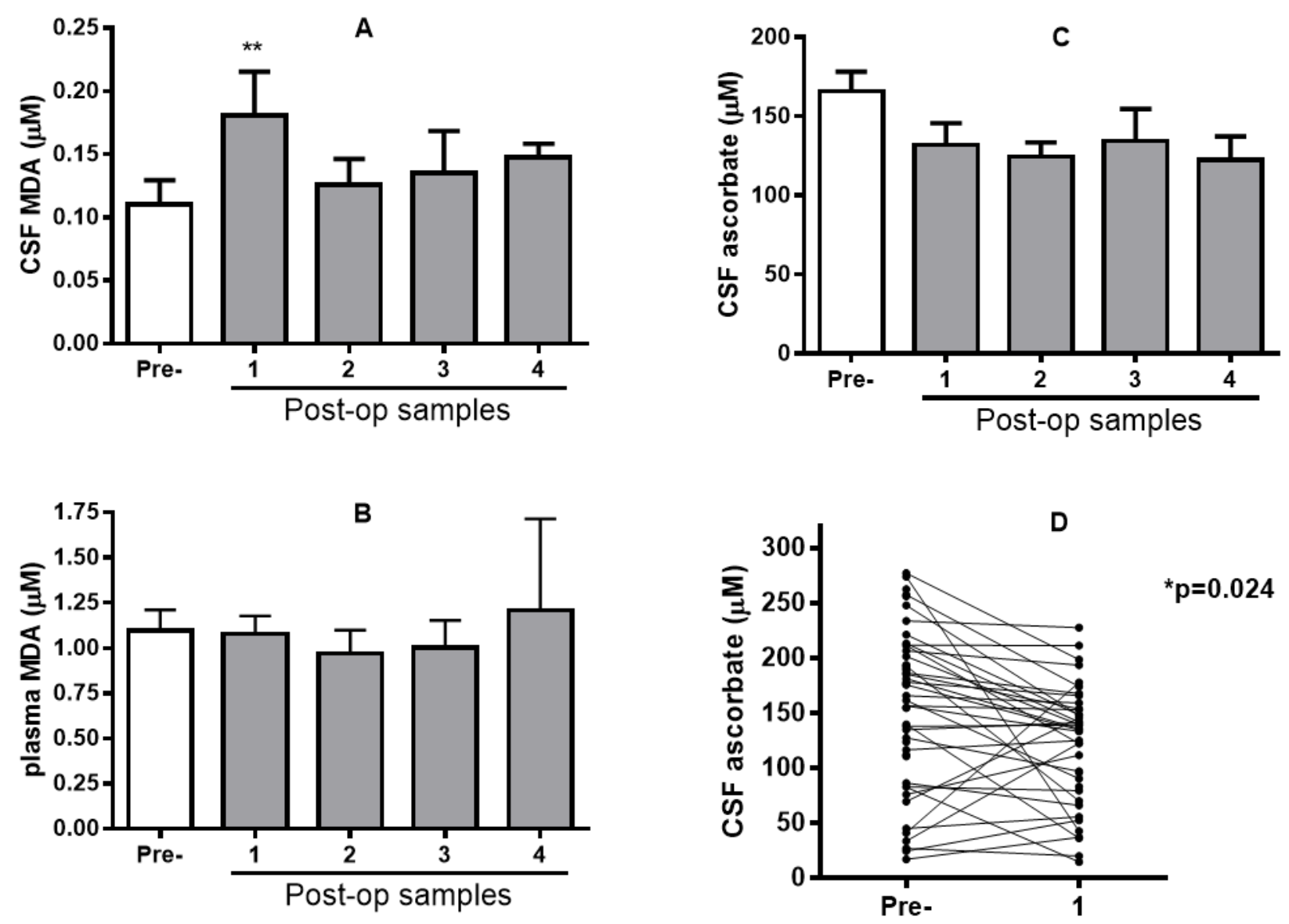

Fig. 1

Malondialdehyde in CSF $a$ and plasma $b$ before and after tumor removal. $P=0.002$ compared with pre-debulking. Data are compared by paired t test; ascorbate in CSF before and after tumor removal; $\mathrm{c}$ all time points; $d$ paired pre-op and post-op samples. $\mathrm{P}=0.024$ compared with pre-debulking compared by paired t-test . 
Perioperative Glucose Levels
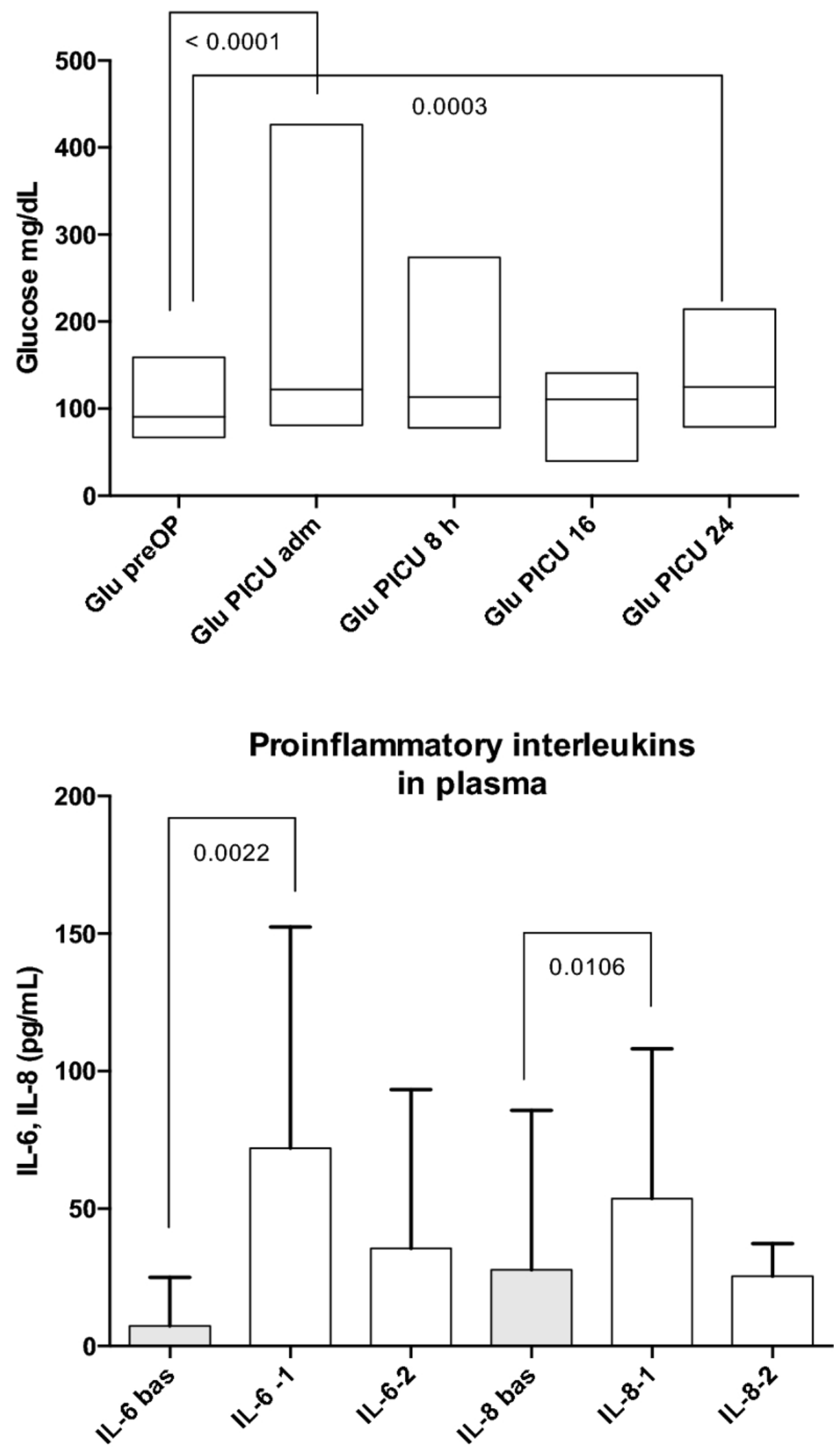

Fig. 2

Perioperative glucose levels $(\mathrm{mg} / \mathrm{dL})$ a and plasma proinflammatory interleukins (IL-6 and IL-8, $\mathrm{pg} / \mathrm{mL}$ ) $\mathrm{b}$ in the study population. 


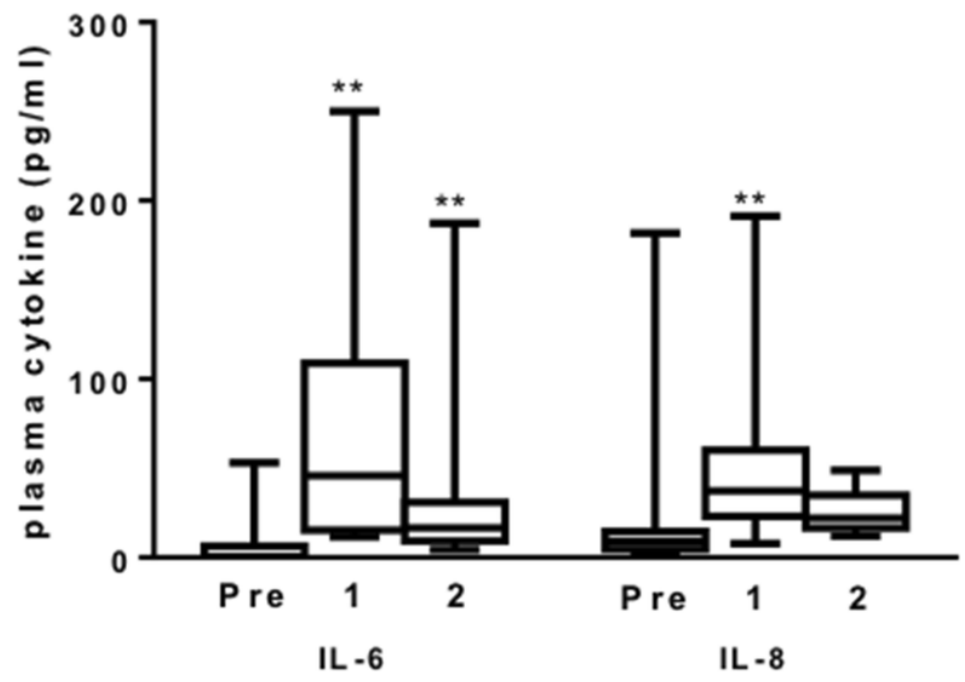

Fig. 3

Plasma IL- 6 and IL-8 before and after tumor removal. P = 0.0039 compared with preoperative values (Wilcoxon matched-pairs test).
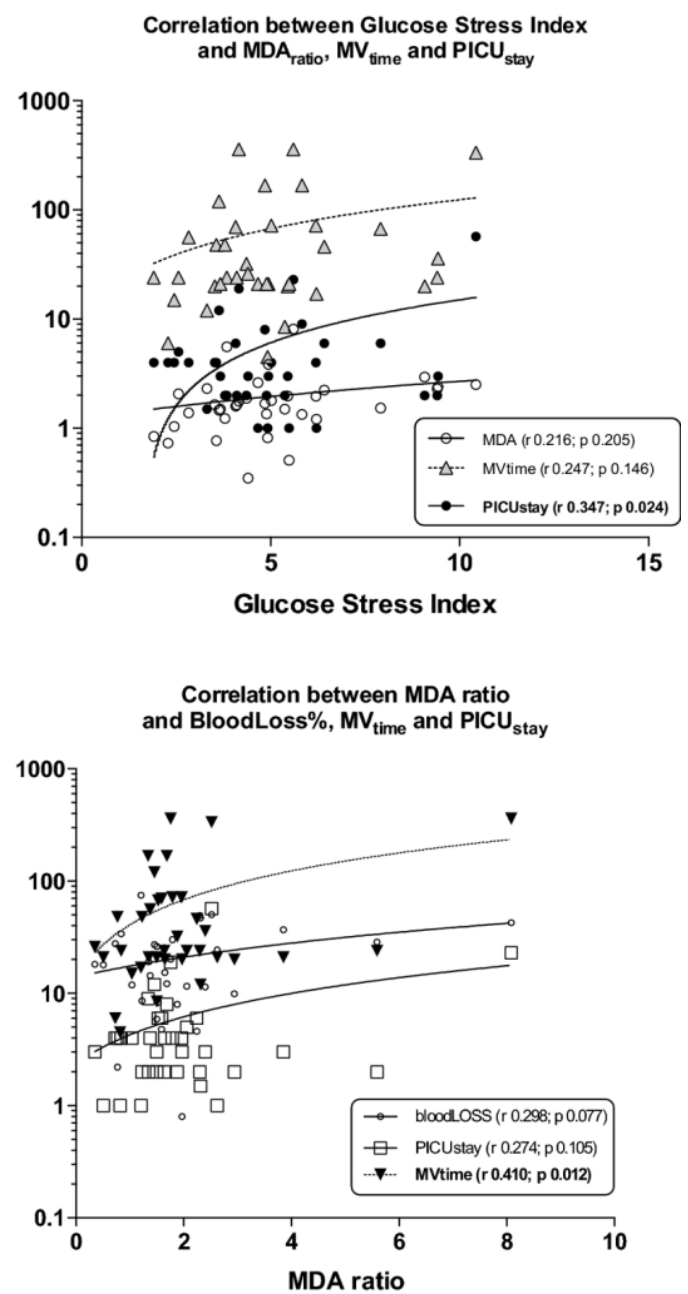

Fig. 4

Correlation between calculated glucose stress index and MDAratio, MVtime, and PICU stay in the study population a; correlation between MDAratio and blood loss (\%) MVtime and PICU stay in the same study population b. MDAratio malondialdehyde ratio,MVtime mechanical ventilation time,PICU stay pediatric intensive care unit stay 


\section{REFERENCE}

1. Deletis $V$, Sala $F$. The role of intraoperative neurophysiology in the protection or documentation of surgically induced injury to the spinal cord. Ann N Y Acad Sci. 2001;939:137-44.

2. Piastra M, Di Rocco C, Caresta E, et al. Blood loss and short-term outcome of infants undergoing brain tumour removal. J Neurooncol. 2008;90:191-200.

3. Borg A, Kirkman MA, Choi D. Endoscopic endonasal anterior skull base surgery: a systematic review of complications over the past 65 years. World Neurosurg. 2016;95:383-91.

4. Jadhav V, Solaroglu I, Obenaus A, et al. Neuroprotection against surgically induced brain injury. Surg Neurol. 2007;67:15-20.

5. Sherchan $\mathrm{P}$, Kim $\mathrm{CH}$, Zhang JH. Surgical brain injury and edema prevention. Acta Neurochir. 2013;118:129-33.

6. Caresta E, Pierro A, Chowdhury M, et al. Oxidation of intravenous lipid in infants and children with systemic inflammatory response syndrome and sepsis. Pediatr Res. 2007;61:228-32.

7. Omaye ST, Turnbull JD, Sauberlich HE. Selected methods for the determination of ascorbic acid in animal cells, tissues, and fluids. Methods Enzymol. 1979;62:3-11.

8. O'Shaughnessy DF, Atterbury C, Bolton MP, et al. Guidelines for the use of fresh-frozen plasma, cryoprecipitate and cryosupernatant. Br J Haematol. 2004;126:11-28.

9. Piastra M, Pizza A, Tosi F, et al. Validation of the Glycemic Stress Index in pediatric neurosurgical intensive care. Neurocrit Care. 2017;26:388-92.

10. Pietrini D, Di Rocco C, Di Bartolomeo R, et al. No-glucose strategy influences posterior cranial fossa tumors' postoperative course: introducing the Glycemic Stress Index. J Neurooncol. 2009;93:361-8.

11. Ayer RE, Zhang JH. Oxydative stress in subarachnoid hemorrhage: significance in acute brain injury and vasospasm. Acta Neurochir Suppl. 2008;104:33-41.

12. Srivastava R, Lohokare R, Prasad R. Oxidative stress in children with bacterial meningitis. J Trop Pediatr. 2013;59:305-8.

13. Kastenbauer $\mathrm{S}$, Koedel $\mathrm{U}$, Becker BF, et al. Oxidative stress in bacterial meningitis in humans. Neurology. 2002;58:186-91.

14. Bayir H, Kagan VE, Tyurina YY, et al. Assessment of antioxidant reserves and oxidative stress in cerebrospinal fluid after severe traumatic brain injury in infants and children. Pediatr Res. 2002;51:571-8.

15. Cristofori L, Tavazzi B, Gambin R, et al. Early onset of lipid peroxidation after human traumatic brain injury: a fatal limitation for the free radical scavenger pharmacological therapy? J Investig Med. 2001;49:450-8.

16. Lewen A, Matz P, Chan PH. Free radical pathways in CNS injury. J Neurotrauma. 2000;17:87190.

17. Inci S, Ozcan OE, Kilinç K. Time-level relationship for lipid peroxidation and the protective effect of alpha-tocopherol in experimental mild and severe brain injury. Neurosurgery. 1998;43:3305.

18. Cirak B, Rousan N, Kocak A, Palaoglu O, Palaoglu S, Kilic K. Melatonin as a free radical scavenger in experimental head trauma. Pediatr Neurosurg. 1999;31(6):298-301.

19. Bayır H, Adelson PD, Wisniewski SR, et al. Therapeutic hypothermia preserves antioxidant defenses after severe traumatic brain injury in infants and children. Crit Care Med. 2009;37:689-95.

20. Zengin E, Atukeren P, Kokoglu E, et al. Alterations in lipid peroxidation and antioxidant status in different types of intracranial tumors within their relative peritumoral tissues. Clin Neurol Neurosurg. 2009;111:345-51. 
21. Pervaiz S, Clement MV. Tumor intracellular redox status and drug resistance - serendipity or a causal relationship? Curr Pharm Des. 2004;10:1969-77.

22. Yilmaz $\mathrm{N}$, Dulger $\mathrm{H}$, Kiymaz $\mathrm{N}$, et al. Lipid peroxidation in patients with brain tumor. Int J Neurosci. 2006;116:937-43.

23. Bujok G, Dyaczyńska-Herman A, Jendryczko A, et al. Concentration of malonic dialdehyde in the cerebrospinal fluid as a measure of the intensity of lipid peroxidation processes in intracranial hypertension in small children. Childs Nerv Syst. 1996;12:97-9.

24. Spentzas T, Escue JE, Patters AB, et al. Brain tumor resection in children: neurointensive care unit course and resource utilization. Pediatr Crit Care Med. 2010;11:718-22.

25. Veluyatham PK, et al. Oxidative stress-associated hypertension in surgically induced brain injury patients: effects of $\beta$-blocker and angiotensin-converting enzyme inhibitor. J Surg Res. 2013;179:125-31. 